\title{
EDUCACIÓN SEDENTARIA CONOCIMIENTO, PODER Y TECNOCRACIA
}

\author{
Vania Ramírez \\ RESUMEN: \\ La sociedad actual demanda y asigna al docente un rol protagónico en la mejora de la calidad \\ de la educación, esto trae consigo volver a remirar sus propias concepciones educativas en \\ relación a la formación y práctica docente, ya que ésta condiciona, limita y a la vez nos \\ entrega nuestras propias prácticas de libertad. El texto pretende relacionar las construcciones \\ tecnócratas de la educación con las concepciones de conocimiento y poder intercaladas \\ desde una mirada de la educación globalizadora. A su vez, más allá de una mirada crítica a \\ estas acepciones el escrito se orientará a establecer al ejercicio docente reflexivo visualizado \\ como un camino de superación de las prácticas descritas anteriormente.
}

Palabras clave: Globalización, Educación tecnócrata, Conocimiento, Reflexión Docente

\section{SEDENTARY EDUCATION: KNOWLEDGE, POWER AND TECHNOCRACY}

\begin{abstract}
:
Today's society demands and assigns to the teacher and a role in improving the quality of education, this brings back Remiro to their own conceptions regarding the educational training and teaching practice, as it affects us, limits us and also gives us our own practice of freedom. The text seeks to relate the constructions of education technocrats with the knowledge and concepts embedded power from the gaze of a global education. In turn, beyond a critical look at these meanings are written on the exercise aimed at establishing reflective teaching seen as a way of overcoming the practices described above.
\end{abstract}

Keywords: Globalization, Education technocrat, Knowledge, Teaching Reflection

\footnotetext{
* Profesora Educación General Básica Universidad Católica de Chile. Docente Adjunto Universidad Cardenal Raúl Silva Henríquez. Estudiante Magíster en Educación, Universidad Academia Humanismo Cristiano. Email: varamirop@gmail.com
} 


\section{Educación globalizada y tecnócrata}

La globalización es un proceso que podemos definir como la extensión del capitalismo, una disciplina económica que atraviesa las ciencias sociales en general y en este sentido, la educación ha sido uno de los frentes más "intervenidos". Así, la globalización no solo se relaciona con distinciones económicas sino también relaciones de nuevas formas de configuración y que van estableciendo diferentes especialidades y nuevas formas de organización del tiempo (Canclini, 1999).

La globalización posee sus propias dinámicas, una explosión de identidades adscriptivas o comunitaristas basadas en el sexo, la edad, la religión, una “verdad” (Garretón, 200I). Todo este sistema estructurado genera exclusión de las diversidades de sujetos que son catalogados como "anormales". Así, la globalización es un proceso desigual e inequitativo, que produce segmentaciones y segregaciones, debido a que establece cómo y qué hacer para conseguir el producto final, por medio de medidas. En el caso de la educación, medidas ministeriales que se proyectan a los centros educativos, definiendo qué es un docente, qué debe hacer y cómo.

La globalización como ente extensor del capitalismo, traspasa las diferentes fronteras, espacios territoriales y sociales que imprimen el sello hacia la economía, la sociedad y el conocimiento y las diversas formas de relacionarnos con nosotros mismos y con los demás.

La escuela se ha vuelto desde hace años, un espacio en el cual se depositan ciertas dinámicas sociales con el fin de mantener el orden social como también para producir cambios. En el caso de la globalización la escuela ha sido un espacio de (re)producción y orden social, insertando ciertas pautas y directrices a seguir que solo representan las clasificaciones o castas de la sociedad, permitiendo por ejemplo la visualización de la educación como un eje económico y no como un aspecto social y de cambio. No es azaroso que las prácticas educativas se encuentren con nuevos discursos emanados de la economía. Cada vez más las instituciones y centros educativos 
se relacionan con discursos tales como "competencias", "capital humano", "niveles de desempeño", "mapas de progreso", etc. Así, la educación se forma como mecanismo de poder, instrumento que permite controlar las conductas personales e institucionales para encauzar hacia el objetivo de la economía y educación global.

Estos controles se han nutrido de las dimensiones teóricas de la educación, las cuales han dado como resultado un sinnúmero de medidas y pautas para conseguir una finalidad específica. En este sentido se ha privilegiado los métodos y técnicas, como una garantía que otorga la seguridad al hacer profesional, y se deja de lado las reflexiones de las prácticas pedagógicas como un espacio de investigación, sistematización y elaboración de conceptualizaciones (Abraham, 2004).

Esta consecución de objetivos preestablecidos se plasma en los diversos manuales y programas que se trazan para el control y la eficiencia social, produciendo demandas por parte de los docentes en la entrega de estas directrices y en las demandas de los futuros docentes en la entrega de estas "recetas" como un conocimiento de urgencia para ejercer la profesión docente, la cual reduce el complejo fenómeno de la educación a la relación enseñanza-aprendizaje y focaliza su atención en la calidad de las metodologías de enseñanza, otorgando componentes de cientificidad a través del planeamiento y su evaluación (Nervi, 2007).

Esta tecnocracia educativa limita a los profesionales educativos y nos dirige hacia una mirada educativa que en primer lugar se centra en esta concepción de enseñanza- aprendizaje como centro de lo educativo, además de eliminar del campo educativo las relaciones entre el conocimiento y la cultura. De esta forma la educación se transforma en un terreno administrativo y de control.

Lo anterior implica que la escuela sea un espacio para la producción y reproducción de servicios y demandas; en donde "un" conocimiento se vuelve esencial y se instaura como eje primordial, atribuyéndole a este espacio características de vaciador, un contenedor de competencias y 
conocimientos que no responden a las particularidades de cada sujeto, y que sobre todo se ha transformado a los hombres y mujeres en un cúmulo de cualidades de "desempeño" y "progreso".

La competencia y el individualismo son valores esenciales de la educación globalizada, que ha sustituido a los actores educativos: padres, madres y familia por instituciones financieras que promueven estos principios.

Como producto de lo anterior, las reformas educacionales se ven permeadas por la producción, el control de la calidad, las competencias, la selección, los sistemas de progresos entre otros, favoreciendo la privatización de la educación, se construye una educación bombardeada de negociaciones, transacciones económicas y humanas del derecho a educar. La educación se cosifica y pierde el sentido de "lo que somos ahora mismo", en este sentido la globalización ha intervenido el tiempo y el espacio, el ahora que no fluctúa, sino que simplemente es y debe ser así. De cierta manera las instituciones forman para tener respuesta a un todo dominado y controlado por el marco curricular impuesto, en donde las propias concepciones institucionales juegan un papel de poder y dominación.

Lo que sucede en el sistema escolar (visualizando al aula), es una especie de eco sobre lo que sucede a nivel sociocultural, incluso un eco de la formación docente inicial, estructurándose el conocimiento y las dinámicas sociales en una construcción de identidades generalizables, prácticas institucionales discusivas y en el accionar, que disciplinan las esperanzas, deseos y expectativas, plasmados en los programas de formación de profesores y en las prácticas de aula” (Popkewitz, I994).

La educación pareciese ser una esfera subalterna de la globalización, y el poder que impregna legítima o no las prácticas de las y los docentes, produciendo lineamientos normalizadores de la educación, segmentándola y transformándola en una educación para "unos(as)" y para "otros(as)". 
Una separación entre los unos(as) (lo local) y los otros(as) (lo global) y sus interacciones establecen relaciones separatistas entre lo global y lo local, y desde esta perspectiva se construyen políticas públicas a los sujetos, estableciendo una confrontación entre las formas de representación.

La educación tecnócrata que es llevada a cabo por instituciones, diversos actores, sistemas públicos y privados, crean un cúmulo de pautas que caen abruptamente cuando estas pautas estipuladas no se relacionan con lo emergente, en donde no existe una relación con un fin, sino más bien con un proceso complejo, que introduce a un sujeto complejo y que no se relaciona con un ideal de sociedad, en este sentido la educación tecnócrata y las escuelas son colaboradoras eficaces en la reproducción de desigualdades, injusticias y prejuicios. De esta forma, el mundo de lo teórico pasa a ser visto, desde la óptica de quienes desempeñan su trabajo en los centros y aulas escolares, como un conocimiento que posee una capacidad de amenaza del status y conocimiento práctico de las profesoras y profesores (Nervi, 2007).

El y la docente se convierte en un/a técnica/o que debe realizar un cierto tipo de actividad, en donde los poderes políticos y gubernamentales se basan en un poder fáctico, una entidad que construye decisiones propias al margen de la democracia (Garretón, 200I) desvinculándose los intereses colectivos y sociales, produciendo para ciertos grupos una mayor calidad educativa y para otros una mayor desigualdad o incluso una negación de la educación, considerando que ésta se construye a partir de un grupo elitista.

La tecnocracia normaliza la educación, y tiende a homogeneizar a las personas que intervienen en el proceso educativo (estudiantes, docentes, directivos). ¿Dónde quedan las subjetividades de los sujetos?, ¿Y para qué se realiza esta normalización?, para que el sistema funcione y de esta forma la continuidad de su propio beneficio.

Todo esto conlleva, a pensar que las políticas están desarrolladas a "prueba de docentes" en donde se ha generalizado a los y las docentes, 
además, de la generalización de las y los estudiantes. Una educación autómata que se basa en: ¿qué es necesario saber?, ¿qué es necesario saber hacer? y ¿cuán bien se debe hacer? o ¿cuán bien se está haciendo?; y frente a estas preguntas, desarrolla dimensiones en las cuales el y la docente debe desenvolver su práctica.

\section{Conocimiento y poder}

La educación tecnócrata se encuentra entrelazada con los conceptos de conocimiento y poder, entregando un "tipo" de conocimiento que implica un "tipo" de poder en el accionar educativo. De esta forma el conocimiento educativo ha estado relacionado al poder quedándose en una suerte de enunciado bidireccional, es decir, todo conocimiento posee un poder en sí y todo poder posee un tipo de conocimiento determinado, determinando el cómo se conoce, qué se conoce y a través de qué medios se conoce. De alguna forma delimita y coarta el conocimiento. Es así como construye el poder dentro de estas prácticas educacionales.

Ahora, es necesario avanzar desde esta relación que está impuesta y es imposible de desaglutinar. Para ello es necesario reconfigurar esta relación a partir de los propios contextos, complejizar y abrir las brechas, en donde el conocimiento puede recartografiarse, reterritorializarse y descentrarse, para reescribir las fronteras y coordenadas de una política cultural de oposición (Giroux, 2003). Una ampliación de las posibilidades.

El conocimiento educativo, y como señala Apple y King, no es simplemente un problema metódico (¿qué debe ser construido como conocimiento?), no es simplemente un problema técnico (¿cómo organizamos el conocimiento?), ni finalmente tampoco, es puramente un problema psicológico (¿cómo conseguimos que los alumnos aprendan?), es más bien el estudio del conocimiento educativo, es un estudio de la ideología.

Complejizar el conocimiento (y su poder) de la educación nos permitiría abrir la brecha de la desigualdad, pero a su vez nos permite 
considerar el currículo desde una perspectiva compleja orientando la (re)construcción de cada espacio bajo sus propios terrenos, en donde la contextualización y la interpretación sean enunciados permanentes y en constante dinamismo, interrogando sobre la finalidad de la escuela, cómo se produce, selecciona, distribuye y legitima el conocimiento (Abraham, 2002). Así, el conocimiento no es una entidad que se acepta sino que se problematiza, ampliando las fronteras de lo técnico.

Una negación a la complejidad del conocimiento instaurado en lo educativo, conllevaría a un estancamiento del propio sujeto impregnándolo de formas únicas de mirar, conocer, sentir, hablar, incluso una única forma de amar.

La construcción del conocimiento orientado por las demandas institucionales y expresado en los diversos objetivos implica "formas de conocimiento, usos lingüísticos, relaciones sociales y valores que implican selecciones y exclusiones particulares a partir de la cultura general, en donde legitima formas particulares de la vida social" (Giroux, 1990).

Estas representaciones son discursos, enunciados que están empapados de intereses ideológicos, políticos y sociales, representaciones mentales construidas a partir del lenguaje; narrativas que involucran visiones de mundo, expresadas a través de reglas y relaciones entre los sujetos que se plasman en orientaciones particulares, valores e intereses, visiones de cómo es el poder, la autoridad y el conocimiento representado (Britzman, 1990).

Todo lo anterior, la globalización, el capital humano, la homogeneización, exclusión de la educación y la desigualdad se puede denominar como un campo educativo sedentario, instaurada sobre la base de una sociedad estática e inmóvil, en donde la educación es concebida bajo el eje reproductor de conocimientos que alguien o un grupo de personas han catalogado como conocimientos "verdaderos" y promueven un discurso, una representación de lo "real" que se produce a través de las instituciones universitarias, entendidas como 
un espacio en donde lo definido como "real" es construido, la verdad se (re) produce y el poder se ejecuta (Britzman, 1990).

Si pensamos la formación docente desde una concepción sedentaria, secular, entonces podríamos relacionarla con una identidad de estudiantes, profesores, directivos, educadores que poseen las mismas características, en donde la representatividad o el pensar se regulan bajo parámetros normativos.

De esta forma pareciese que existiera una identidad educativa única y verdadera de la significación del docente, y el lenguaje de la multiplicidad y pluralización de los discursos de la realidad se ubican fuera de este proyecto. Entonces nos encontramos con un manejo del poder, de la construcción del conocimiento a partir de lo dado, una identidad educativa que se construye a partir de estas representaciones de la realidad y que se va constituyendo a partir de la objetivización de las características que ciertas personas creen que significan ser docente.

Así, la práctica de los propios profesores y profesoras es considerada en bastantes ocasiones como no relevante, monótona y mecánica; se ignora el valor como fuente de conocimiento profesional y se reduce a la esfera de lo privado, sin posibilidades de ser compartido con los compañeros y compañeras (Jackson, I994).

\section{La posibilidad de la reflexión}

Si planteamos un enfoque distinto, un giro en la construcción y reconstrucción de la educación, que se encuentre fuera del mercantilismo global, fuera de lo técnico en la educación, involucra considerar la educación como un proceso de desplazamientos constantes y no estancados. Significa dimensionar una estructuración y re-estructuración variable, en donde se considere la heterogeneidad del sujeto, un cuestionamiento constante a los espacios de referencia y de interacción con el otro, cambiando desde un enfoque de caracterización educativa fija hacia uno que cuestione el "cómo, desde qué, por quién y para qué" de ésta, que nos permita "saber quiénes 
somos, quiénes no somos y quiénes podríamos ser" (Britzman, I990). Además, significa considerar la educación como un proceso complejo, en el cual intervienen una serie de experiencias.

Este giro es posible, gracias a la reflexión que nace como un modelo para contrarrestar las prácticas técnicas, debido a este desajuste explicitado anteriormente, a este encuentro con una realidad compleja y contextualizada por las características dinámicas de la sociedad. La reflexión como "una dimensión esencial, idiosincrásica de cualquier profesional que desempeña trabajos prácticos, tal como ocurre con el colectivo docente" (Jackson, I994). Sin este componente reflexivo el y la docente se enfrenta a realizar sus prácticas al amparo de la intuición.

La reflexión se nutre de las propias dinámicas y formación de los sujetos, que implica volver a mirarlo, de esta forma el educador deberá tener en cuenta la individualidad que está presente en cada uno de sus estudiantes, procurando por todos los medios que esté a su alcance favorecer su desarrollo. Evitando un aplicación de predominancia impersonal y uniforme (Durkheim, 1976). En otras palabras, someter la variedad de prácticas educativas a la reflexión, caracterizada por un accionar en la acción que tome giros de acuerdo a los dinámicos contextos en los que se sitúa el proceso educativo.

Esta reflexión posee como eje fundamental la consideración de la historicidad de los propios sujetos, con miradas hacia el cambio. Cambio que comienza por los propios agentes educativos que la escuela y la sociedad han designado la función formadora, los docentes, pero que a su vez permite establecer la reflexión como un modo de visualizar la vida y que permite establecer relaciones con los estudiantes, padres, madres, directivos, es decir que sea un eje de la comunidad educativa en general y que a su vez permita un accionar basado en la colaboración y no en la jerarquía.

La reflexión considera tomar en cuenta la multiplicidad de contextos en los cuales se desenvuelve la educación, caracterizados por las dinámicas relaciones entre diversos actores educativos, (re) 
construyendo los significados del accionar educativo. Además, la reflexión contribuiría hacia un camino de independencia de estas leyes tácitas con las cuales se impregna la educación, visualizando a la reflexión como un espacio de democracia y emancipación docente.

Por lo tanto deberíamos imaginar el espacio educativo como un espacio dialógico de interrelaciones en donde se ponen en juego las concepciones relacionales del mundo (Massey, 2005), echando abajo las concepciones esencialistas y deterministas de la educación, un espacio que implique la existencia y reconocimiento de esta multiplicidad. Massey determina este espacio que posea: tiempo de interacción que debería darse bajo la multiplicidad y para ésta deben haber espacios para ello que contengan relaciones existentes y futuras siempre cambiantes.

La reflexión implica valorizar la complejidad del sujeto vivenciada a través de la propia multiplicidad de subjetividades y que se van formulando y reformulando en un encuentro de diversos discursos los cuales convergen o se desencuentran, coexisten, se influyen y entran en conflicto; produciendo complejidades y producto de estas interrelaciones que son de carácter activo y dinámico, se van produciendo las subjetividades, creadoras de una verdad no certera y que por tanto nunca se encuentran finalizados ni concluidos, movilizaciones culturales que permiten el surgimiento de nuevos sujetos e identidades culturales entrelazadas de los cambios y transformaciones en el sistema de representaciones simbólicas tradicional (Amparan, 2000).

\section{Conclusiones}

Como conclusión, es relevante situar la construcción y reconstrucción de la educación como una experiencia que no sea ni determine de forma tajante al sujeto, es decir, que entre las políticas curriculares preescritas y las posiciones en las cuales se adscribe el sujeto, sea concebido como un espacio de reconstrucciones contantes, incluso otorgando una caracterización de dinamismo al proceso educativo. 
A su vez, que este proceso no contenga en sí mismo un resultado, que no posea condicionantes ni apremiantes anteriores, que no se dé por supuesta ni que sea certera. Una educación que pueda incluso trabajar el conflicto como parte de un aprendizaje, más que rechazar y evadir la complejidad de los distintos contextos y dimensiones que cruzan el eje educativo.

En este sentido, el proceso reflexivo no significa una negación de la teoría, sino más bien una nueva forma de ver cómo se relacionan las construcciones entre teoría y práctica, en que ambos se nutren y dialogan entre sí, y que esta relación implica considerar a los actores educativos como agentes activos, que van (re)construyendo los significados que le otorgan a la educación.

Una educación que no posea una finalidad establecida, que vaya más allá del mero acontecimiento, sino más bien que se pregunte por lo que pasa y qué generan las diversas experiencias y conocimientos que la componen. Una experiencia que no se vuelque a establecer solo un análisis del diálogo enunciado, sino más bien que la experiencia hable por sí misma, que nos exprese quienes somos, unos narradores de la experiencia (Britzman, 1990) cargada de sorpresas y contradicciones que posean como objetivo, la creación de espacios que fomenten la multiplicación de placeres, deseos, voces e intereses (Seidman, 1993).

\section{Bibliografía}

Abraham, Mirtha (2000). Análisis de algunas perspectivas teóricas del currículum. Primavera 2000. Apuntes.

(2004). El campo educativo y la pedagogía: desafíos y limitaciones. Apuntes.

Apple, M. y King, N. (I990). "Economía política y control de la vida cotidiana”, en Ideología y currículum. Morata, Madrid.

Britzman, D. (1990). "The terrible problem of knowing thyself: towards a poststructuralist view of teacher identity". Journal of Curriculum Theorizing. Chicago, EEUU.

Canclini, N. (I999). La globalización imaginada. Paidós, Barcelona. 
Durkheim, E. (1976). Educación como socialización. Salamanca.

Garretón, M. (200I). Cambios sociales, actores y acción colectiva en América Latina. Capítulo III: Cambios sociales y nuevo contexto histórico. CEPAL.

Giroux, H. (2003). "La pedagogía de los límites en la era del postmodernismo”, en Pedagogía y política de la esperanza. Amorrortu, Buenos Aires.

Jackson, P. (1994). La vida en las aulas. Morata, Madrid.

Popkewit, T. (1994). "Política, conocimiento y poder: algunas cuestiones para el estudio de las reformas educativas”, en Revista de Educación. Vol. Sept.Dic. No. 305 (pp. 103 a I37). Centro de Publicaciones, Madrid.

Nervi, L. (2007). ¿Existe la pedagogía? Hacia la construcción del saber pedagógico. Editorial Universitaria, Santiago de Chile.

Talburt, S. (2005). "Política de identidad, respuesta institucional y negociación cultural: significados de la creación de una oficina universitaria de gays y lesbianas", en Pensando Queer. Sexualidad, cultura y educación. Editorial Graó. España. 